\title{
Rhodium-Catalyzed Asymmetric Conjugate Additions of Boronic Acids to Enones with DIPHONANE: a Novel Chiral Bisphosphine Ligand
}

Koen Vandyck, Bavo Matthys, Mario Willen, Koen Robeyns ${ }^{\S}$, Luc Van Meervelt ${ }^{\S}$ and Johan Van der Eycken*

Laboratory for Organic and Bioorganic Synthesis, Department of Organic Chemistry, Ghent University, Krijgslaan 281 (S.4), B-9000 Gent (Belgium).

$\S$ Biomolecular Architecture, Department of Chemistry, K. U. Leuven,

Celestijnenlaan 200F, B-3001 Leuven (Heverlee) (Belgium).

Corresponding Author: mailto:Johan.Vandereycken@UGent.be

\section{SUPPORTING INFORMATION}




\section{Table of contents}

General experimental methods .

Synthesis of bistriflate $( \pm)-2$

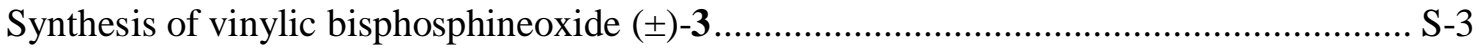

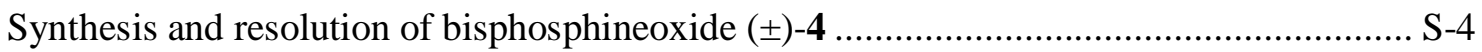

HPLC Chromatogram (Chiralcel OD-H column), ( $( \pm)-\mathbf{4},(1 R, 2 R, 4 R, 5 R)-\mathbf{4}$,

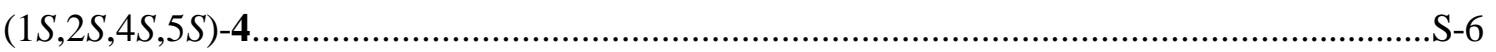

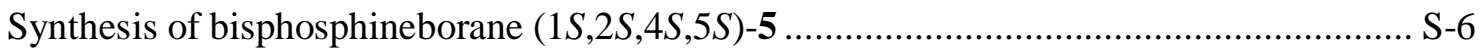

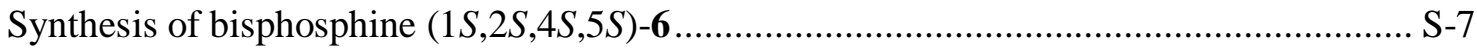

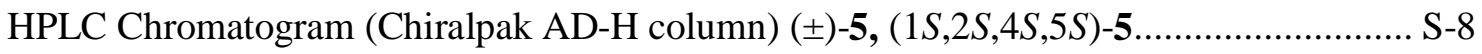

Typical experimental procedure for the $\mathrm{Rh}(\mathrm{I}) /(1 S, 2 S, 4 S, 5 S)$-DIPHONANE

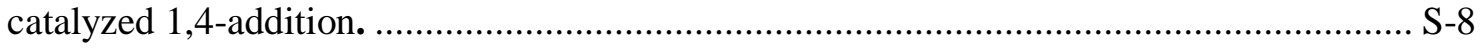

Determination of the relative and absolute configuration of alcohol 14 Aa.................. S-10

${ }^{1} \mathrm{H}$ and ${ }^{13} \mathrm{C}$ NMR spectra of compounds $2,3,4,5,6$, complex 4:8, 14Aa and

its epimer 15Aa and 13Cd.

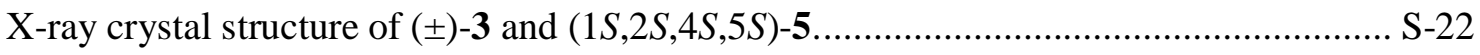


All reactions were carried out under argon atmosphere in dry solvents under anhydrous conditions, unless otherwise noted. Reagents were purchased and used without purification, unless otherwise stated. $(R, R)$ and $(S, S)$-Acid 8 were synthesized in two steps from commercial dibenzyltartrate according to reference 12 c. 2,5-Norbornadione was synthesized according to reference 6 . The indicated $R_{\mathrm{f}}$-values are for Macherey-Nagel SIL UV254 TLCplates. Flash chromatography was carried out with Merck Kieselgel 60, 30-75 $\mu \mathrm{m} .{ }^{1} \mathrm{H}-\mathrm{NMR}$, ${ }^{13} \mathrm{C}$-NMR and ${ }^{31} \mathrm{P}-\mathrm{NMR}$ were recorded on a Bruker Avance 300 or a Bruker AM 500 spectrometer as indicated, with chemical shifts reported in ppm relative to the residual solvent signal. The IR-spectra are recorded with a Perkin-Elmer 160 FT-IR spectrometer. Mass spectra were recorded with a Hewlett-Packard 5988A mass spectrometer. Analytical chiral HPLC and LC/MS were recorded on an Agilent 1100 system.

Synthesis of bistriflate $( \pm)-2$. Diketone $( \pm)-1(100 \mathrm{mg}, 0.806 \mathrm{mmol})$ was dissolved in dry THF (5.5 mL) and the mixture was cooled to $-78{ }^{\circ} \mathrm{C}$. KHMDS $(4.2 \mathrm{~mL}, 0.5 \mathrm{M}$ in toluene, 2.1 mmol, 2.6 base-equiv) was slowly added and after $1 \mathrm{~h} \operatorname{PhNTf}_{2}$ (753 mg, $2.11 \mathrm{mmol}, 2.6$ equiv), dissolved in dry THF (2 mL) at room temperature, was directly added to the reaction mixture through a double tipped needle at $-78{ }^{\circ} \mathrm{C}$. The reaction was stopped after $3 \mathrm{~h}$ of further stirring at $-78{ }^{\circ} \mathrm{C}$ by quenching with a saturated solution of $\mathrm{NH}_{4} \mathrm{Cl}(10 \mathrm{~mL})$. After warming to room temperature, the mixture was extracted with pentane $(2 \times 20 \mathrm{~mL})$ and the organic layers were washed with another portion of saturated aqueous $\mathrm{NH}_{4} \mathrm{Cl}(10 \mathrm{~mL})$. Drying with $\mathrm{MgSO}_{4}$, filtration and removal of the volatiles resulted in a crude mixture which was purified by chromatography over silicagel using gradient elution: pentane/toluene 99:1 to pentane/ $\mathrm{Et}_{2} \mathrm{O} 98: 2$ to pentane/ $\mathrm{Et}_{2} \mathrm{O} 80: 20$ resulting in $222 \mathrm{mg}(0.572 \mathrm{mmol}, 71 \%)$ bistriflate $( \pm)-2$ as a colourless liquid. $\mathrm{R}_{\mathrm{f}}: 0.41\left(c-\mathrm{Hex} \mathrm{Et}_{2} \mathrm{O} 9: 1\right) .{ }^{1} \mathrm{H}-\mathrm{NMR}: \delta_{\mathrm{H}}\left(500 \mathrm{MHz}, \mathrm{CDCl}_{3}\right): 2.62$ $(2 \mathrm{H}, \mathrm{t}, \mathrm{J}=1.8 \mathrm{~Hz}), 3.53(2 \mathrm{H}, \mathrm{m}), 6.51\left(2 \mathrm{H}, \mathrm{dd}(\right.$ app. t), J = 2.4, $2.4 \mathrm{~Hz})$ ppm. ${ }^{13} \mathrm{C}-\mathrm{NMR}: \delta_{\mathrm{C}}$ $\left(125.7 \mathrm{MHz}, \mathrm{CDCl}_{3}\right): 50.3(\mathrm{CH}), 73.1\left(\mathrm{CH}_{2}\right), 118.4(\mathrm{q}, \mathrm{J}=321 \mathrm{~Hz}, \mathrm{C}), 123.7(\mathrm{CH}), 168.1(\mathrm{C})$ ppm. IR (KBr, thin film): 1631, 1610, 1430, 1294, 1282, 1251, 1214, 1139, 1074, 1027, 935, 919, 884, 871, $845 \mathrm{~cm}^{-1}$. MS m/z (rel. intensity \%): $388\left(\mathrm{M}^{+}, 13\right), 227$ (11), 163 (3), 122 (9), 105 (12), 94 (23), 77 (100).

Synthesis of vinylic bisphosphine oxide ( \pm )-3. A mixture of bistriflate $( \pm)-2(1.90 \mathrm{~g}, 4.89$ mmol) and DIPEA (5.4 mL, $31.0 \mathrm{mmol}, 6$ equiv) was dissolved in toluene (50 mL) in a 100 $\mathrm{mL}$ flask. The mixture was deoxygenated (3 freeze-pump-thaw cycles) and $\mathrm{HPPh}_{2}(2.24 \mathrm{~mL}$, 
$12.9 \mathrm{mmol}, 2.5$ equiv), ( \pm )-BINAP (160 $\mathrm{mg}, 0.26 \mathrm{mmol}, 5 \mathrm{~mol} \%)$ and $\mathrm{Pd}(\mathrm{OAc})_{2}(58 \mathrm{mg}$, $0.26 \mathrm{mmol}, 5 \mathrm{~mol} \%$ ) were consecutively added. The mixture was stirred for $16 \mathrm{~h}$ at $45{ }^{\circ} \mathrm{C}$ after which it was poured in a $500 \mathrm{~mL}$ erlenmeyer flask containing $\mathrm{CH}_{2} \mathrm{Cl}_{2}(100 \mathrm{~mL})$. To this solution, $\mathrm{H}_{2} \mathrm{O}_{2}(5 \mathrm{~mL}, 35 \mathrm{w} / \mathrm{w} \%)$ was added and stirring was continued for $15 \mathrm{~min}$ at room temperature. After the addition of $\mathrm{H}_{2} \mathrm{O}(80 \mathrm{~mL})$, the organic layer was separated and the water layer was extracted with $\mathrm{CH}_{2} \mathrm{Cl}_{2}(3 \times 75 \mathrm{~mL})$. The organic layers were washed with aqueous $1 \mathrm{~N} \mathrm{HCl}(80 \mathrm{~mL})$ and dried with $\mathrm{Na}_{2} \mathrm{SO}_{4}$. After filtration and removal of the volatiles, the crude mixture was purified by chromatography over silica gel applying gradient elution: acetone $/ \mathrm{CH}_{2} \mathrm{Cl}_{2} /$ pentane $70 / 15 / 15 ; \mathrm{CH}_{2} \mathrm{Cl}_{2} / \mathrm{MeOH} 97 / 3 ; \mathrm{CH}_{2} \mathrm{Cl}_{2} / \mathrm{MeOH} 95 / 5$. In this way, $2.24 \mathrm{~g}$ vinylic phosphine-oxide ( \pm )-3 $(4.55 \mathrm{mmol}, 93 \%)$ was isolated. The product could be recrystallized from acetonitrile. Single crystals suitable for X-ray diffraction studies were grown by slow evaporation of a $\mathrm{CH}_{3} \mathrm{CN}$ solution of ( \pm )-3 (See page 22 for X-ray diffraction results) $\mathrm{R}_{\mathrm{f}}:$ 0,20 $\left(\mathrm{CH}_{2} \mathrm{Cl}_{2} / \mathrm{MeOH}\right.$ 95:5). mp 207-208 ${ }^{\circ} \mathrm{C}\left(\mathrm{CH}_{3} \mathrm{CN}\right) .{ }^{1} \mathrm{H}-\mathrm{NMR}: \delta_{\mathrm{H}}(500 \mathrm{MHz}$, $\mathrm{CDCl}_{3}$ ): 2.29 (2H, app. s), 4.08 (2H, app. s), 7.09 (2H, ddd (app. dt), J = 8.7, 1.8, $1.8 \mathrm{~Hz}$ ), 7.40-7.48 (8H, m), 7.49-7.58 (8H, m), 7.60-7.67 (4H, m) ppm. ${ }^{13} \mathrm{C}-\mathrm{NMR}: \delta_{\mathrm{C}}(75.5 \mathrm{MHz}$, $\left.\mathrm{CDCl}_{3}\right)$ : $54.0(\mathrm{CH}, \mathrm{dd}, \mathrm{J}=10.4,12.6 \mathrm{~Hz}), 73.1\left(\mathrm{CH}_{2}, \mathrm{t}, \mathrm{J}=4.4 \mathrm{~Hz}\right), 128.6(\mathrm{CH}, \mathrm{d}, \mathrm{J}=13.2$ Hz), 131.1 (C, d, J = 107.0 Hz), 131.4 (C, d, J = 107.0 Hz), $131.3(\mathrm{CH}, \mathrm{d}, \mathrm{J}=10.0 \mathrm{~Hz}), 131.5$ $(\mathrm{CH}, \mathrm{d}, \mathrm{J}=10.0 \mathrm{~Hz}), 132.0(\mathrm{CH}, \mathrm{d}, \mathrm{J}=2.7 \mathrm{~Hz}), 148.3(\mathrm{C}, \mathrm{dd}, \mathrm{J}=109.8,2.2 \mathrm{~Hz}), 158.2(\mathrm{CH}$, d, J $=11.0 \mathrm{~Hz})$ ppm. ${ }^{31} \mathrm{P}-\mathrm{NMR}: \delta_{\mathrm{P}}\left(121.4 \mathrm{MHz}, \mathrm{CDCl}_{3}\right): 23.4$ ppm. ES-MS: $493[\mathrm{M}+\mathrm{H}]^{+} . \mathrm{MS}$ $m / z$ (rel. intensity \%): $492\left(\mathrm{M}^{+}, 64\right), 462$ (3), 415 (3), 367 (6), 291 (52), 266 (77), 228 (26), 201 (82), 184 (81), 77 (100), IR (KBr, thin film): 3049, 1553, 1433, 1274, 1186, 1119, 1102 , $997,751,724 \mathrm{~cm}^{-1}$.

Synthesis and resolution of bisphosphine oxide ( \pm )-4. Phosphine oxide ( \pm )-3 (4.07 g, 8.26 mmol) was dissolved in methanol $(400 \mathrm{~mL})$ and was shaken with $\mathrm{Pd} / \mathrm{C}(4.0 \mathrm{~g}, 10 \mathrm{w} / \mathrm{w} \%)$ in a Parr reactor under $4 \mathrm{~atm} \mathrm{H}_{2}$ for $45 \mathrm{~h}$. The palladium catalyst was removed by filtration over celite and was washed with $\mathrm{MeOH}$. The obtained crude mixture weighed $4.19 \mathrm{~g}$. The racemic compound could be recrystallized from acetone. The crude mixture was applied in the resolution step. $\mathrm{R}_{\mathrm{f}}$ : $0.11\left(\mathrm{CH}_{2} \mathrm{Cl}_{2} / \mathrm{MeOH}\right.$ 95:5). mp: 162-164 ${ }^{\circ} \mathrm{C}$ (acetone). ${ }^{1} \mathrm{H}-\mathrm{NMR}: \delta_{\mathrm{H}}(500$ $\left.\mathrm{MHz}, \mathrm{CD}_{3} \mathrm{OD}\right):$ 1.53-1.62 (2H, m), 1.66-1.72 (2H, m), 2.61 (2H, app. s), 2.78-2.83 (2H, m), $2.91(2 \mathrm{H}, \mathrm{ddd}, \mathrm{J}=7.8,12.9,16.0 \mathrm{~Hz}), 7.46-7.58(12 \mathrm{H}, \mathrm{m}), 7.74-7.85(8 \mathrm{H}, \mathrm{m}) \mathrm{ppm} .{ }^{13} \mathrm{C}-\mathrm{NMR}$ : $\delta_{\mathrm{C}}\left(125.7 \mathrm{MHz}, \mathrm{CD}_{3} \mathrm{OD}\right): 24.7\left(\mathrm{CH}_{2}, \mathrm{~d}, \mathrm{~J}=3.4 \mathrm{~Hz}\right), 41.3(\mathrm{CH}, \mathrm{d}, \mathrm{J}=5.9 \mathrm{~Hz}), 42.3(\mathrm{CH}, \mathrm{d}, \mathrm{J}=$ $78.8 \mathrm{~Hz}), 44.6\left(\mathrm{CH}_{2, \mathrm{t}} \mathrm{t}, \mathrm{J}=15.2 \mathrm{~Hz},\right), 129.8(\mathrm{CH}, \mathrm{d}, \mathrm{J}=11.9 \mathrm{~Hz}), 129.9(\mathrm{CH}, \mathrm{d}, \mathrm{J}=11.9 \mathrm{~Hz})$, 
$132.0(\mathrm{CH}, \mathrm{d}, \mathrm{J}=8.5 \mathrm{~Hz}), 132.1(\mathrm{CH}, \mathrm{d}, \mathrm{J}=8.5 \mathrm{~Hz}), 132.9(\mathrm{CH}), 134.6(\mathrm{C}, \mathrm{d}, \mathrm{J}=97.5 \mathrm{~Hz})$, $135.2(\mathrm{C}, \mathrm{d}, \mathrm{J}=96.6 \mathrm{~Hz})$ ppm. ${ }^{31} \mathrm{P}-\mathrm{NMR}: \delta_{\mathrm{P}}\left(121.4 \mathrm{MHz}, \mathrm{CDCl}_{3}\right)$ : 31.6 ppm. ES-MS: 497 $[\mathrm{M}+\mathrm{H}]^{+} . \mathrm{MS} \mathrm{m} / z$ (rel. intensity \%): $496\left(\mathrm{M}^{+}, 9\right), 418$ (7), 295 (100), 229 (76), 201 (30), 77 (40). IR (KBr, thin film): 3055, 2962, 2879, 1437, 1188, $1118,751,721,700 \mathrm{~cm}^{-1}$. Conditions for HPLC: Chiralcel OD-H column, solvent: $n$-hexane/EtOH (95:5), flow rate = $1 \mathrm{~mL} / \mathrm{min}, \mathrm{T}=35^{\circ} \mathrm{C}$, retention times for $(1 R, 2 R, 4 R, 5 R)-\mathbf{4}=20-25 \mathrm{~min}$, for $(1 S, 2 S, 4 S, 5 S)-\mathbf{4}=$ 23-28 min.

In a $100 \mathrm{~mL}$ flask, crude bisphosphine oxide $( \pm)-4(1.312 \mathrm{~g})$ was dissolved in $\mathrm{CHCl}_{3}(40 \mathrm{~mL})$. In a $25 \mathrm{~mL}$ flask, $(R, R)-(2,3-\mathrm{di}[($ phenylamino)carbonyl]tartaric acid (1.026 g, $2.64 \mathrm{mmol})$ was suspended in EtOAc $(10.5 \mathrm{~mL})$. Both solutions were heated in an oilbath of $80{ }^{\circ} \mathrm{C}$. After 5 min, the EtOAc solution was added dropwise to the $\mathrm{CHCl}_{3}$ solution and the $25 \mathrm{~mL}$ flask was washed with EtOAc $(2.5 \mathrm{~mL})$, which was also added to the mixture. After another 5 min at 80 ${ }^{\circ} \mathrm{C}$, the mixture was filtered warm and the filtrate was refluxed for a further $15 \mathrm{~min}$ and slowly cooled. The flask was then cooled to $4{ }^{\circ} \mathrm{C}$ and stored overnight after which the diastereomerically pure $(1 S, 2 S, 4 S, 5 S)-\mathbf{4}-(R, R)-\mathbf{8} 1: 1$ complex was collected on a filter as a white solid (842 mg, $0.952 \mathrm{mmol}, 37 \%$ based on $( \pm)-3)$ and washed with $\mathrm{CHCl}_{3}(30 \mathrm{~mL}) .{ }^{1} \mathrm{H}-$ NMR: $\delta_{\mathrm{H}}\left(500 \mathrm{MHz} ; \mathrm{CD}_{3} \mathrm{OD}\right): 1.52-1.62(2 \mathrm{H}, \mathrm{m}), 1.68$ (2H, app. s), 2.60 (2H, app. s), 2.75$2.83(2 \mathrm{H}, \mathrm{m}), 2.90(\mathrm{ddd}, 2 \mathrm{H}, \mathrm{J}=7.8,12.9,16.0 \mathrm{~Hz}), 5.71(2 \mathrm{H}, \mathrm{s}), 7.03(2 \mathrm{H}$, app. t, J = 7.6 Hz), 7.27 (4H, app. t, J = 7.6 Hz), 7.43 (4H, app. d, J = 7.6 Hz), 7.46-7.59 (12H, m), 7.76-7.82 $(8 \mathrm{H}, \mathrm{m})$ ppm. ${ }^{13} \mathrm{C}-\mathrm{NMR}: \delta_{\mathrm{C}}\left(75.5 \mathrm{MHz}, \mathrm{CD}_{3} \mathrm{OD}\right): 24.7\left(\mathrm{CH}_{2}, \mathrm{~d}, \mathrm{~J}=3.8 \mathrm{~Hz}\right), 41.3(\mathrm{CH}, \mathrm{dd}, \mathrm{J}$ = 1.6, 6.6 Hz), $42.4(\mathrm{CH}, \mathrm{d}, \mathrm{J}=78.5 \mathrm{~Hz}), 44.6\left(\mathrm{CH}_{2}, \mathrm{t}, \mathrm{J}=15.3 \mathrm{~Hz}\right), 73.3(\mathrm{CH}), 120.1(\mathrm{CH})$, $124.4(\mathrm{CH}), 129.7(\mathrm{CH}, \mathrm{d}, \mathrm{J}=11.5 \mathrm{~Hz}), 129.86(\mathrm{CH}, \mathrm{d}, \mathrm{J}=11.5 \mathrm{~Hz}), 129.87(\mathrm{CH}), 132.0$ $(\mathrm{CH}, \mathrm{d}, \mathrm{J}=8.8 \mathrm{~Hz}), 132.1(\mathrm{CH}, \mathrm{d}, \mathrm{J}=8.8 \mathrm{~Hz}), 132.9(\mathrm{CH}, \mathrm{d}, \mathrm{J}=2.2 \mathrm{~Hz}), 134.6(\mathrm{C}, \mathrm{d}, \mathrm{J}=97$ $\mathrm{Hz}), 135.1$ (C, d, J = $97 \mathrm{~Hz}), 139.8$ (C), 154.5 (C), 170.5 (C) ppm. ${ }^{31} \mathrm{P}-\mathrm{NMR}: \delta_{\mathrm{P}}(121.4 \mathrm{MHz}$, $\mathrm{CD}_{3} \mathrm{OD}$ ): 34.3 ppm. IR (KBr): 3404, 3297, 3201, 3137, 3058, 2961, 2880, 2401, 1911, 1735 , 1602, 1542, 1501, 1438, 1314, 1213, 1135, 1121, 1086, 1025, 753, 724, $692 \mathrm{~cm}^{-1}$. $(1 S, 2 S, 4 S, 5 S)-\mathbf{4}-(R, R)-8:[\alpha]_{\mathrm{D}}^{20}=-40(\mathrm{c} 0.75, \mathrm{MeOH})$ The same procedure can be applied with $(S, S)-8:(1 R, 2 R, 4 R, 5 R)-\mathbf{4}-(S, S)-8:[\alpha]_{\mathrm{D}}^{20}=+37(\mathrm{c} 0.74, \mathrm{MeOH})$.

$(1 S, 2 S, 4 S, 5 S)-\mathbf{4}-(R, R)-\mathbf{8}(739 \mathrm{mg}, 0.835 \mathrm{mmol})$ was suspended in $\mathrm{CH}_{2} \mathrm{Cl}_{2}(350 \mathrm{~mL})$ and washed with a $1 \mathrm{M} \mathrm{NaHCO}$ solution $(3 \times 100 \mathrm{~mL})$ followed by $\mathrm{H}_{2} \mathrm{O}(1 \times 100 \mathrm{~mL})$. The organic layer was dried with $\mathrm{Na}_{2} \mathrm{SO}_{4}$, filtered and evaporated. Drying of the product resulted in $390 \mathrm{mg}(1 S, 2 S, 4 S, 5 S)-4(0.785 \mathrm{mmol}, 94 \%)$. The product showed a small optical rotation 
and since small impurities were present it was further transformed and purified as its boraneprotected analogue.
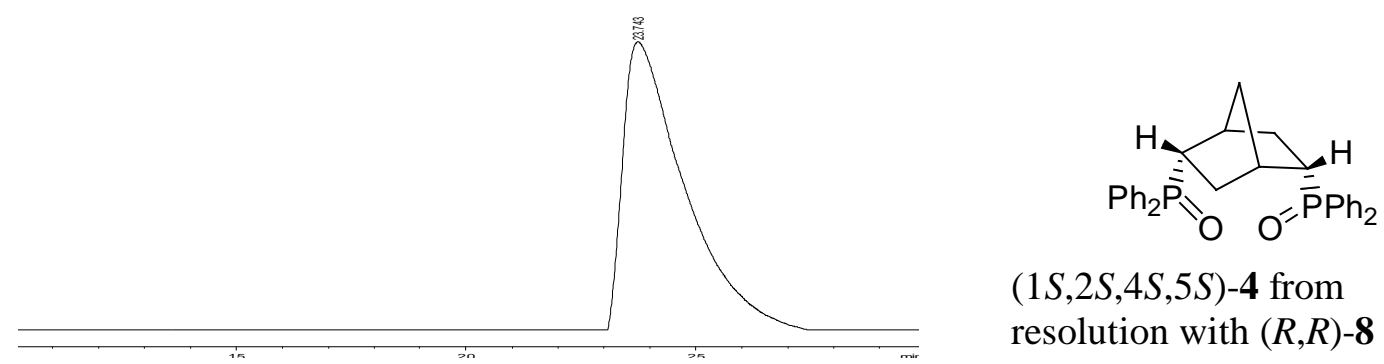

$(1 S, 2 S, 4 S, 5 S)-\mathbf{4}$ from resolution with $(R, R)-\mathbf{8}$
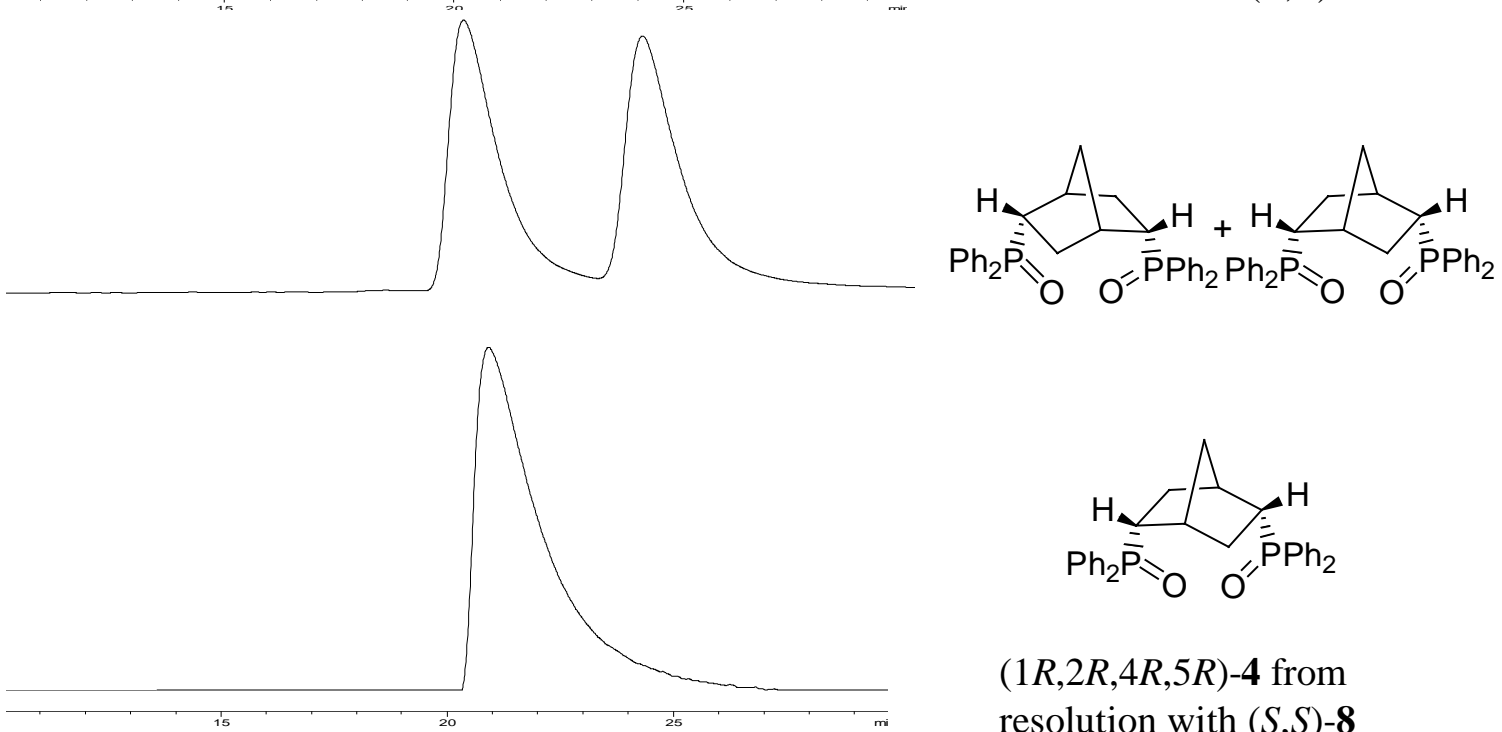

$(1 R, 2 R, 4 R, 5 R)-4$ from resolution with $(S, S)-\mathbf{8}$

Conditions for HPLC: Chiralcel OD-H column, solvent: $n$-hexane/EtOH (95:5), flow rate = $1 \mathrm{~mL} / \mathrm{min}, \mathrm{T}=35^{\circ} \mathrm{C}$, retention times $(1 R, 2 R, 4 R, 5 R)-\mathbf{4}=20-25 \min (1 S, 2 S, 4 S, 5 S)-\mathbf{4}=23-28$ $\min$.

Synthesis of bisphosphineborane (1S,2S,4S,5S)-5. To $(1 S, 2 S, 4 S, 5 S)-4(334.2 \mathrm{mg}, 0.673$ $\mathrm{mmol})$ in toluene $(9.5 \mathrm{~mL})$ in a pressure tube, was added $\mathrm{Ti}\left(\mathrm{O}^{\mathrm{i}} \mathrm{Pr}\right)_{4}(115 \mu \mathrm{L}, 0.390 \mathrm{mmol}, 0.6$ equiv.) and $\mathrm{HSi}(\mathrm{OEt})_{3}(750 \mu \mathrm{L}, 4.06 \mathrm{mmol}, 6$ equiv). After purging with argon the tube was closed and heated to $100^{\circ} \mathrm{C}$ for $2.5 \mathrm{~h}$. The black-brown solution was then cooled to room temperature and $\mathrm{BH}_{3}$.DMS (260 $\mu \mathrm{l}, 2.7 \mathrm{mmol}, 4$ equiv) was added. After $15 \mathrm{~min}$ of stirring, the excess reagent was carefully destroyed with $\mathrm{MeOH}$ and the volatiles were removed. The product was purified by chromatography over silica gel applying a $\mathrm{CH}_{2} \mathrm{Cl}_{2} / n$-hexane $45 / 55$ mixture followed by a 50/50 mixture as eluent resulting in $(1 S, 2 S, 4 S, 5 S)-5(245.3 \mathrm{mg}, 0.498$ mmol, 74\%) as a white solid. Single crystals suitable for X-ray diffraction studies were grown by slow evaporation of a $\mathrm{CH}_{3} \mathrm{CN}$ solution of $(1 S, 2 S, 4 S, 5 S)-5$ (See page 22 for X-ray 
diffraction results) $\mathrm{R}_{\mathrm{f}}: 0.10$ ( $n$-Hexane/ $\mathrm{CH}_{2} \mathrm{Cl}_{2}$ 60:40), mp: decomposition $>190^{\circ} \mathrm{C},{ }^{1} \mathrm{H}-\mathrm{NMR}$ : $\delta_{\mathrm{H}}\left(500 \mathrm{MHz}, \mathrm{CDCl}_{3}\right): 0.60-1.35$ (6H, broad signal), $1.53-1.64(2 \mathrm{H}, \mathrm{m}), 1.68$ (2H, app. s), 2.26 (2H, ddd (app. dt), J = 8.6, 13.3, $13.3 \mathrm{~Hz}), 2.65-2.75$ (4H, m), 7.36-7.48 (12H, m), 7.58$7.69(8 \mathrm{H}, \mathrm{m}) \mathrm{ppm} .{ }^{13} \mathrm{C}-\mathrm{NMR}: \delta_{\mathrm{C}}\left(75.5 \mathrm{MHz}, \mathrm{CDCl}_{3}\right): 25.4\left(\mathrm{CH}_{2}\right.$, dd (app. t), J = 2.7, $\left.2.7 \mathrm{~Hz}\right)$, $40.1(\mathrm{CH}, \mathrm{dd}, \mathrm{J}=2.7,6.6 \mathrm{~Hz}), 40.8(\mathrm{CH}, \mathrm{d}, \mathrm{J}=35.7 \mathrm{~Hz}), 44.2\left(\mathrm{CH}_{2}, \mathrm{t}, \mathrm{J}=12.8 \mathrm{~Hz}\right), 128.61$ $(\mathrm{C}, \mathrm{d}, \mathrm{J}=9.6 \mathrm{~Hz}), 128.66(\mathrm{CH}, \mathrm{d}, \mathrm{J}=9.6 \mathrm{~Hz}), 129.5(\mathrm{C}, \mathrm{d}, \mathrm{J}=54.4 \mathrm{~Hz}), 130.7(\mathrm{CH}, \mathrm{d}, \mathrm{J}=2.2$ Hz), $130.9(\mathrm{CH}, \mathrm{d}, \mathrm{J}=2.2 \mathrm{~Hz}), 131.6(\mathrm{C}, \mathrm{d}, \mathrm{J}=55.4 \mathrm{~Hz}), 132.2(\mathrm{CH}, \mathrm{d}, \mathrm{J}=8.8 \mathrm{~Hz}), 133.1$ $(\mathrm{CH}, \mathrm{d}, \mathrm{J}=8.8 \mathrm{~Hz}) .{ }^{31} \mathrm{P}-\mathrm{NMR}: \delta_{\mathrm{P}}\left(121.4 \mathrm{MHz}, \mathrm{CDCl}_{3}\right) 16.8(\mathrm{~m}) . \mathrm{MS} \mathrm{m} / z$ (rel. intensity \%): 477 (24), 387 (7), 215 (63), 183 (100). IR (KBr, thin film): 2394, 2324, 1479, 1453, 1436, $1134,1103,1060,1021,736,697 \mathrm{~cm}^{-1}$. The enantiomeric purity at this stage was confirmed to be $>99 \%$ ee Conditions for HPLC: Chiralpak AD-H column, solvent: n-hexane/i-PrOH (50:50), flow rate $=1 \mathrm{~mL} / \mathrm{min}, \mathrm{T}=35{ }^{\circ} \mathrm{C}$, retention times: $(1 S, 2 S, 4 S, 5 S)-5=6.0 \mathrm{~min}$, $(1 R, 2 R, 4 R, 5 R)-5=7.8 \mathrm{~min}$. Optical rotation for $(1 S, 2 S, 4 S, 5 S)-5:[\alpha]_{\mathrm{D}}^{20}=+35\left(\mathrm{c} 0.99, \mathrm{CHCl}_{3}\right)$, $[\alpha]_{365}^{20}=+113\left(\mathrm{c} 0.99, \mathrm{CHCl}_{3}\right)$; for $(1 R, 2 R, 4 R, 5 R)-5:[\alpha]_{\mathrm{D}}^{20}=-36\left(\mathrm{c} 0.99, \mathrm{CHCl}_{3}\right),[\alpha]_{365}^{20}=-$ 115 (c $\left.0.99, \mathrm{CHCl}_{3}\right)$.

Synthesis of bisphosphine $(1 S, 2 S, 4 S, 5 S)-6$. A heterogeneous mixture of $(1 S, 2 S, 4 S, 5 S)-5$ (100.6 mg, $0.204 \mathrm{mmol})$ in $\mathrm{EtOH}(3 \mathrm{~mL})$ was deoxygenated applying three freeze-pump-thaw cycles in a pressure tube. The mixture was heated at $80{ }^{\circ} \mathrm{C}$ for $16 \mathrm{~h}$ and after cooling of the homogeneous solution, a white solid precipitated from the solution. Removal of the volatiles and dissolution in a deoxygenated $70 / 30 n$-Hexane $/ \mathrm{CH}_{2} \mathrm{Cl}_{2}$ mixture was followed by filtration over neutral alumina $(8 \mathrm{~mL})$ in a glove box with the same solvent mixture $(50 \mathrm{~mL})$. Removal of the solvent resulted in $90.6 \mathrm{mg}(1 S, 2 S, 4 S, 5 S)-6(0.195 \mathrm{mmol}, 96 \%)$ as a white solid. $\mathrm{R}_{\mathrm{f}}$ : $0.27(n-\mathrm{Hex} / \mathrm{Tol} 50: 50)$; mp $141^{\circ} \mathrm{C}$; optical rotation for $(1 S, 2 S, 4 S, 5 S)-6:[\alpha]_{\mathrm{D}}^{20}=+35(\mathrm{c} 0.54$, $\left.\mathrm{CHCl}_{3}\right),[\alpha]_{365}^{20}=+234\left(\mathrm{c} 0.54, \mathrm{CHCl}_{3}\right) .{ }^{1} \mathrm{H}-\mathrm{NMR}: \delta_{\mathrm{H}}\left(500 \mathrm{MHz}, \mathrm{C}_{6} \mathrm{D}_{6}\right): 1.32(2 \mathrm{H}$, app. s), 1.45-1.55 (2H, m), 2.13-2.18 (2H, m), 2.45 (2H, ddd, J = 6.6, 6.6, $12.9 \mathrm{~Hz}), 2.48-2.56(2 \mathrm{H}$, m), 7.02-7.16 $(12 \mathrm{H}, \mathrm{m})$ 7.40-7.48 $(4 \mathrm{H}, \mathrm{m}), 7.55-7.63(4 \mathrm{H}, \mathrm{m})$ ppm. ${ }^{13} \mathrm{C}-\mathrm{NMR}: \delta_{\mathrm{C}}(75.5 \mathrm{MHz}$ , $\left.\mathrm{C}_{6} \mathrm{D}_{6}\right): 30.2\left(\mathrm{CH}_{2}, \mathrm{t}, \mathrm{J}=21.2 \mathrm{~Hz}\right) 41.0(\mathrm{CH}, \mathrm{t}, \mathrm{J}=7.7 \mathrm{~Hz}), 41.4(\mathrm{CH}, \mathrm{dd}, \mathrm{J}=4.3,5.7 \mathrm{~Hz})$, $42.5\left(\mathrm{CH}_{2}, \mathrm{t}, \mathrm{J}=4.3 \mathrm{~Hz}\right), 128.4-128.7(\mathrm{CH}, \mathrm{m}), 133.3-134.1(\mathrm{CH}, \mathrm{m}), 139.3-140.6(\mathrm{C}, \mathrm{m})$ ppm. ${ }^{31} \mathrm{P}-\mathrm{NMR}: \delta_{\mathrm{P}}\left(121.4 \mathrm{MHz}, \mathrm{C}_{6} \mathrm{D}_{6}\right):-3.0 \mathrm{ppm}$. IR (KBr, thin film): 3068, 3048, 2952, $2865,1584,1479,1445,1432,1384,1256,1153,1093,1067,1026,741,695 \mathrm{~cm}^{-1} \mathrm{MS} \mathrm{m} / \mathrm{z}$ 
(rel. intensity \%): 464 (M+12), 387 (12), 279 (52), 213 (100), 199 (18), 183 (96), 152 (13), $133(22)$
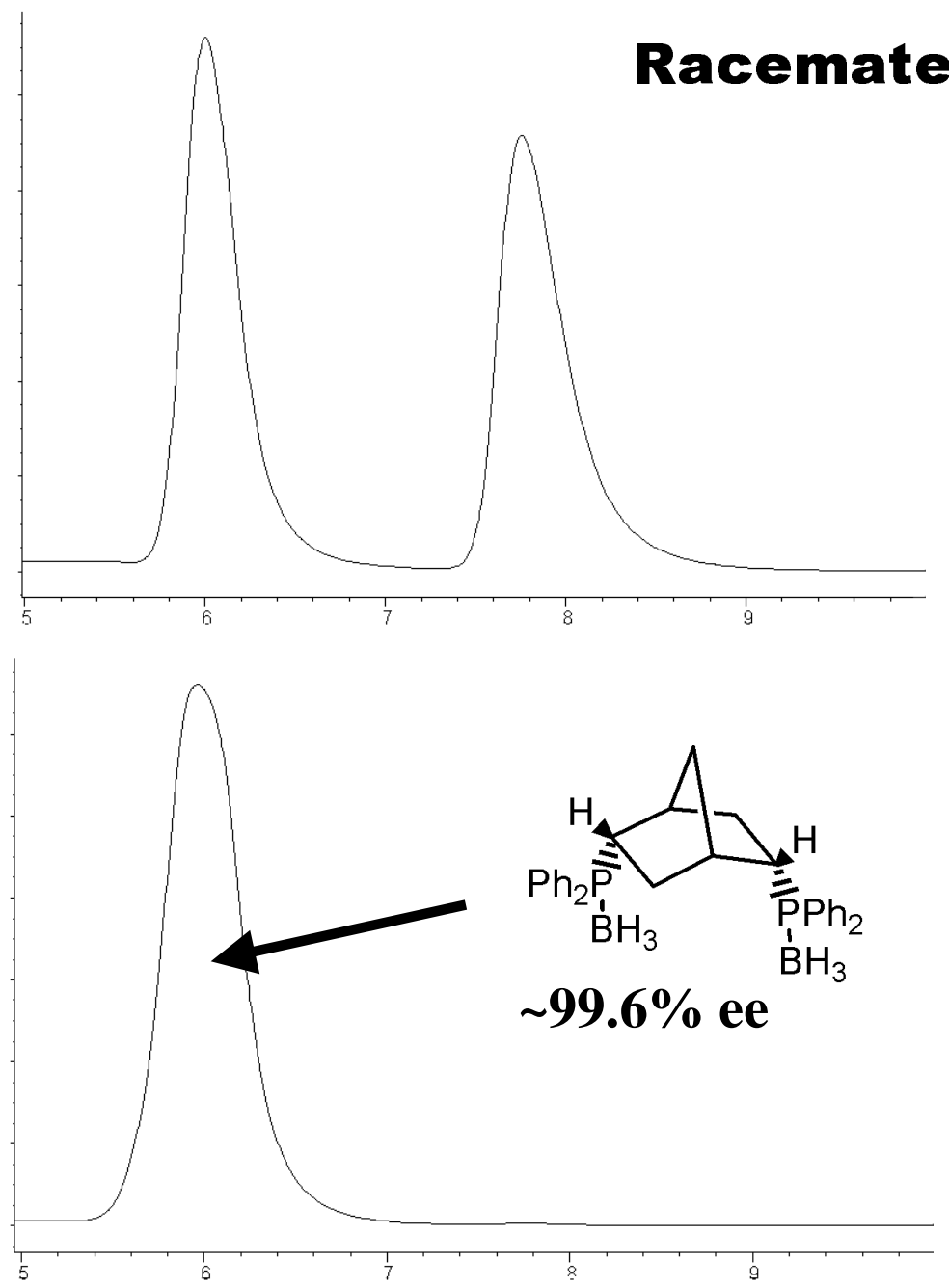

Typical experimental procedure for the $\mathrm{Rh}(\mathrm{I}) /(1 S, 2 S, 4 S, 5 S)$-DIPHONANE catalyzed

\section{1,4-addition.}

To $\left[\mathrm{RhCl}\left(\mathrm{C}_{2} \mathrm{H}_{4}\right)_{2}\right]_{2}(1.6 \mathrm{mg}, 0.00823 \mathrm{mmol} \mathrm{Rh}, 2 \times 1.0 \mathrm{~mol} \% \mathrm{Rh}),(2 S, 5 S)$-DIPHONANE (4.3 mg, $0.00923 \mathrm{mmol}, 2 \times 1.1 \mathrm{~mol} \%)$ and $\mathrm{KOH}(71 \mathrm{mg}, 1.27 \mathrm{mmol})$ in a reaction tube under argon, degassed 1,4-dioxane $(1.2 \mathrm{~mL})$ and degassed $\mathrm{H}_{2} \mathrm{O}(220 \mu \mathrm{L})$ were added. The mixture was stirred for $30 \mathrm{~min}$ at room temperature, after which it was separated into two equal parts with a syringe. Each part was transferred to a tube containing a boronic acid (2.07 mmol, 5 equiv. $)$ and an enone $(0.413 \mathrm{mmol})$ under argon. The original flask and the syringe 
were rinced with degassed 1,4-dioxane $(1 \mathrm{~mL})$ and this solution was added to each reaction tube until the total amount of solvent reached $1.18 \mathrm{~mL}$ in each reaction tube. The reaction tubes were sealed and stirred under argon at $50{ }^{\circ} \mathrm{C}$. After the indicated time both mixtures were cooled down and filtered over a plug of silica gel with a 1:2 EtOAc/n-Hexane mixture. Evaporation of the vollatiles was followed by chromatography on silica gel (hexane/ $\mathrm{Et}_{2} \mathrm{O}$ ).

Conditions for HPLC with chiral stationary phase: flow rate $=1 \mathrm{~mL} / \mathrm{min}, \mathrm{T}=35^{\circ} \mathrm{C}$ :

3-phenylcyclohexanone: Chiralpak AD-H column, solvent: $n$-hexane/i-PrOH (99:1), retention times: $(3 S)=15.1 \mathrm{~min},(3 R)=17.2 \mathrm{~min}$. 3-(1-naphthyl $)$ cyclohexanone: Chiralpak AD-H column, solvent: $n$-hexane/ $i-\mathrm{PrOH}(98: 2)$, retention times: $(-)=12.1 \mathrm{~min},(+)=13.5$ min. 3-(4-trifluoromethylphenyl)cyclohexanone: Chiralpak AD-H column, solvent: $n$-hexane/i-PrOH (99:1), retention times: $(+)=14.0 \mathrm{~min},(-)=15.1 \mathrm{~min}$. 3-(4-methoxyphenyl)cyclohexanone: Chiralcel OD-H column, solvent: n-hexane/iPrOH (98:2), retention times: $(+)=13.0 \mathrm{~min},(-)=13.6 \mathrm{~min}$. The enantiomeric excess was confirmed by ${ }^{13} \mathrm{C}-\mathrm{NMR}$ analysis of its $(+)-(R, R)$-DPEN derived aminal. ${ }^{19}$ 3-(o-tolyl)cyclohexanone: Chiralcel OD-H column, solvent: $\mathrm{n}$-hexane/iPrOH (99:1), retention times: $(+)=20.0 \mathrm{~min},(-)=21.8 \mathrm{~min}$. 3-phenyl-2-nonanone Chiralcel OD-H column, solvent: $n$-hexane/i-PrOH (99:1), retention times: $(+)=9.7 \mathrm{~min},(-)=10.3 \mathrm{~min} .3-$ phenylcyclopentanone: Chiralpak AD-H column, solvent: $n$-hexane $/ i$-PrOH (99:1), retention times: $(+)=14.8 \mathrm{~min},(-)=15.5 \mathrm{~min}$ 3-phenylcycloheptanone Chiralcel OD-H column, solvent: $n$-hexane/i-PrOH (98:2), retention times: $(-)=11.7 \mathrm{~min},(+)=13.3 \mathrm{~min}$

3-(1-naphthyl)cycloheptenone $\mathrm{R}_{\mathrm{f}}: 0.27$ (pentaan/Et ${ }_{2} \mathrm{O}$ 80/20); ${ }^{1} \mathrm{H}-\mathrm{NMR}: \delta_{\mathrm{H}}(500 \mathrm{MHz}$, $\left.\mathrm{CDCl}_{3}\right)$ : 1.59-1.71 $(1 \mathrm{H}, \mathrm{m}), 1.73-1.95(2 \mathrm{H}, \mathrm{m}), 2.02-2.21(2 \mathrm{H}, \mathrm{m}), 2.21-2.33(1 \mathrm{H}, \mathrm{m}), 2.68-$ $2.74(2 \mathrm{H}, \mathrm{m}), 2.79$ (1H, ddd (app. dt), J = 2.1, 2.1, $14.2 \mathrm{~Hz}), 3.02(1 \mathrm{H}, \mathrm{dd}, \mathrm{J}=11.7,14.2 \mathrm{~Hz})$, $3.73(1 \mathrm{H}, \mathrm{m}), 7.35(1 \mathrm{H}$, app. d, J = $8.0 \mathrm{~Hz}), 7.44(1 \mathrm{H}$, app. t, $\mathbf{J}=8.0 \mathrm{~Hz}), 7.49(1 \mathrm{H}, \mathrm{ddd}, \mathrm{J}=$ 0.9, 6.8, 8.0 Hz), $7.55(1 \mathrm{H}, \mathrm{ddd}, \mathrm{J}=1.1,6.8,8.6 \mathrm{~Hz}), 7.73(1 \mathrm{H}$, app. d, J = 8.0 Hz), $7.87(1 \mathrm{H}$, app. d, J $=8.0 \mathrm{~Hz}), 8.06\left(1 \mathrm{H}\right.$, app. d, J = 8.6 Hz) ppm. ${ }^{13} \mathrm{C}-\mathrm{NMR}: \delta_{\mathrm{C}}\left(75.5 \mathrm{MHz}, \mathrm{CDCl}_{3}\right)$ : $24.3\left(\mathrm{CH}_{2}\right), 29.5\left(\mathrm{CH}_{2}\right), 37.1(\mathrm{CH}), 38.7\left(\mathrm{CH}_{2}\right), 44.0\left(\mathrm{CH}_{2}\right), 50.8\left(\mathrm{CH}_{2}\right), 122.4(\mathrm{CH}), 122.8$ $(\mathrm{CH}), 125.5(\mathrm{CH}), 125.5(\mathrm{CH}), 126.2(\mathrm{CH}), 126.9(\mathrm{CH}), 129.1(\mathrm{CH}), 130.6(\mathrm{C}), 134.0(\mathrm{C})$, 142.6 (C), 213.6 (C) ppm. ES-MS m/z (rel. intensity \%): 221.1 (100) $\left[\mathrm{M}-\mathrm{H}_{2} \mathrm{O}+\mathrm{H}\right]^{+}, 239.1$ (19) $[\mathrm{M}+\mathrm{H}]^{+}, 256.1(19)\left[\mathrm{M}+\mathrm{NH}_{4}\right]^{+}, 261.1$ (17) $[\mathrm{M}+\mathrm{Na}]^{+}$; IR (KBr, thin film): 2926, 2850, 1697, 1597, 1511, 1504, 1452, 1445, 1395, 1346, 1267, 1235, 1194, 1080, 1023, 796, $778 \mathrm{~cm}^{-1}$

\footnotetext{
${ }^{19}$ Alexakis, A.; Frutos, J. C.; Mangeney, P. Tetrahedron: Asymm. 1993, 4, 12, 2431-2434
} 
Chiralpak AD-H column, solvent: $n$-hexane/ $i$-PrOH (99:1), retention times: $(-)=19.3$ min, $(+)$ $=21.2 \mathrm{~min}$.

DETERMINATION OF THE RELATIVE AND ABSOLUTE CONFIGURATION OF $\underline{\text { ALCOHOL 14Aa }}$

$(1 R, 3 R)-\mathbf{1 4 A a},[\alpha]_{\mathrm{D}}^{20}=+20.7\left(\mathrm{c} 0.41, \mathrm{CHCl}_{3}, 89 \%\right.$ ee $),[\alpha]_{365}^{20}=+78.0\left(\mathrm{c} 0.41, \mathrm{CHCl}_{3}, 89 \%\right.$ ee)

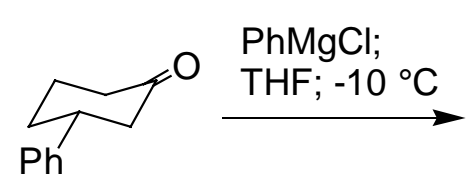

$( \pm)-13 \mathrm{Aa}$

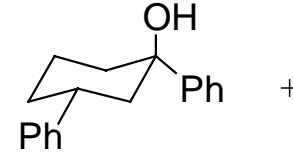

$( \pm)-14 \mathrm{Aa}$ $58 \%$

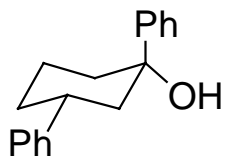

$( \pm)-15 \mathrm{Aa}$ $41 \%$

\section{Scheme 1.}

To ( \pm -13Aa $(30.0 \mathrm{mg}, 0.172 \mathrm{mmol})$ in $\operatorname{dry} \operatorname{THF}(1.5 \mathrm{~mL})$ at $-10{ }^{\circ} \mathrm{C}, \mathrm{PhMgCl}(180 \mu \mathrm{L}, 2.0 \mathrm{M}$ in THF, $0.360 \mathrm{mmol}, 2$ equiv) was added. After $0.5 \mathrm{~h}$, the mixture was poured into saturated $\mathrm{NaHCO}_{3}(5 \mathrm{~mL})$ and extracted with EtOAc (2x $\left.15 \mathrm{~mL}\right)$. Drying with $\mathrm{Na}_{2} \mathrm{SO}_{4}$, filtration and removal of the volatiles resulted in a crude oil mixture which was purified by chromatography over silica gel with a 80/20 $n$-hexane/ $\mathrm{Et}_{2} \mathrm{O}$ eluent. The first eluted alcohol was $( \pm)-\mathbf{1 4 A a}(25.2$ $\mathrm{mg}, 0.100 \mathrm{mmol}$, oil, 58\%) followed by the more slowly eluting ( \pm )-15Aa $(17.8 \mathrm{mg}, 0.071$ mmol, white solid, 41\%). ( \pm )-14Aa: $\mathrm{R}_{\mathrm{f}} 0.48$ (EtOAc/n-hexane 1:2), ${ }^{1} \mathrm{H}-\mathrm{NMR}: \delta_{\mathrm{H}}(500 \mathrm{MHz}$, $\left.\mathrm{CDCl}_{3}\right): 1.53\left(\mathrm{H}_{4}{ }^{\mathrm{ax}}\right.$, dddd (app. dq), J = 3.4, 12.5, 12.5, $\left.12.5 \mathrm{~Hz}\right), 1.95\left(\mathrm{H}_{2}{ }^{\mathrm{ax}}\right.$, dd (app. t), 12.5, $12.5 \mathrm{~Hz}), 1.82-2.07(6 \mathrm{H}, \mathrm{m}), 3.17\left(\mathrm{H}_{3}{ }^{\mathrm{ax}}\right.$, dddd, J = 3.4, 3.4, 12.5, $\left.12.5 \mathrm{~Hz}\right), 7.19\left(\mathrm{H}_{\mathrm{para}}\right.$, tt, J =

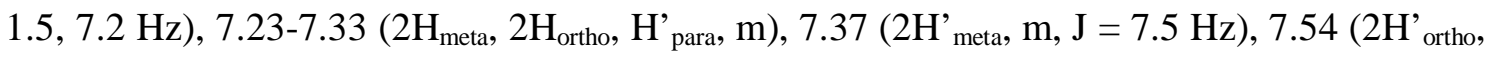
m, J $=7.5 \mathrm{~Hz})$ ppm. ${ }^{13} \mathrm{C}-\mathrm{NMR}: \delta_{\mathrm{C}}\left(75.5 \mathrm{MHz}, \mathrm{CDCl}_{3}\right): 22.3\left(\mathrm{CH}_{2}\right), 33.2\left(\mathrm{CH}_{2}\right), 38.3\left(\mathrm{CH}_{2}\right)$, $39.6(\mathrm{CH}), 46.6\left(\mathrm{CH}_{2}\right), 73.8(\mathrm{C}), 124.4(\mathrm{CH}), 126.0(\mathrm{CH}), 126.9(\mathrm{CH}), 127.0(\mathrm{CH}), 128.3$ (CH), $128.4(\mathrm{CH}), 146.7$ (C), 149.3 (C) ppm. MS m/z (rel. intensity \%): 234 (66), 209 (74), 133 (31), 120 (61), 105 (100), 91 (58), 77 (69). IR (KBr, thin film): 3554, 3422, 3084, 3059, 3026, 2932, 2850, 1600, 1493, 1445, 1256, 1129, 1069, 982, $754 \mathrm{~cm}^{-1}$. Conditions for HPLC, Chiralcel OD-H column, flow rate $=1 \mathrm{~mL} / \mathrm{min}, \mathrm{T}=35^{\circ} \mathrm{C}$, solvent: $n$-hexane $/ i-\mathrm{PrOH}(95: 5)$, retention times: $(1 S, 3 S)=10.0 \mathrm{~min},(1 R, 3 R)=15.9 \mathrm{~min}$. 


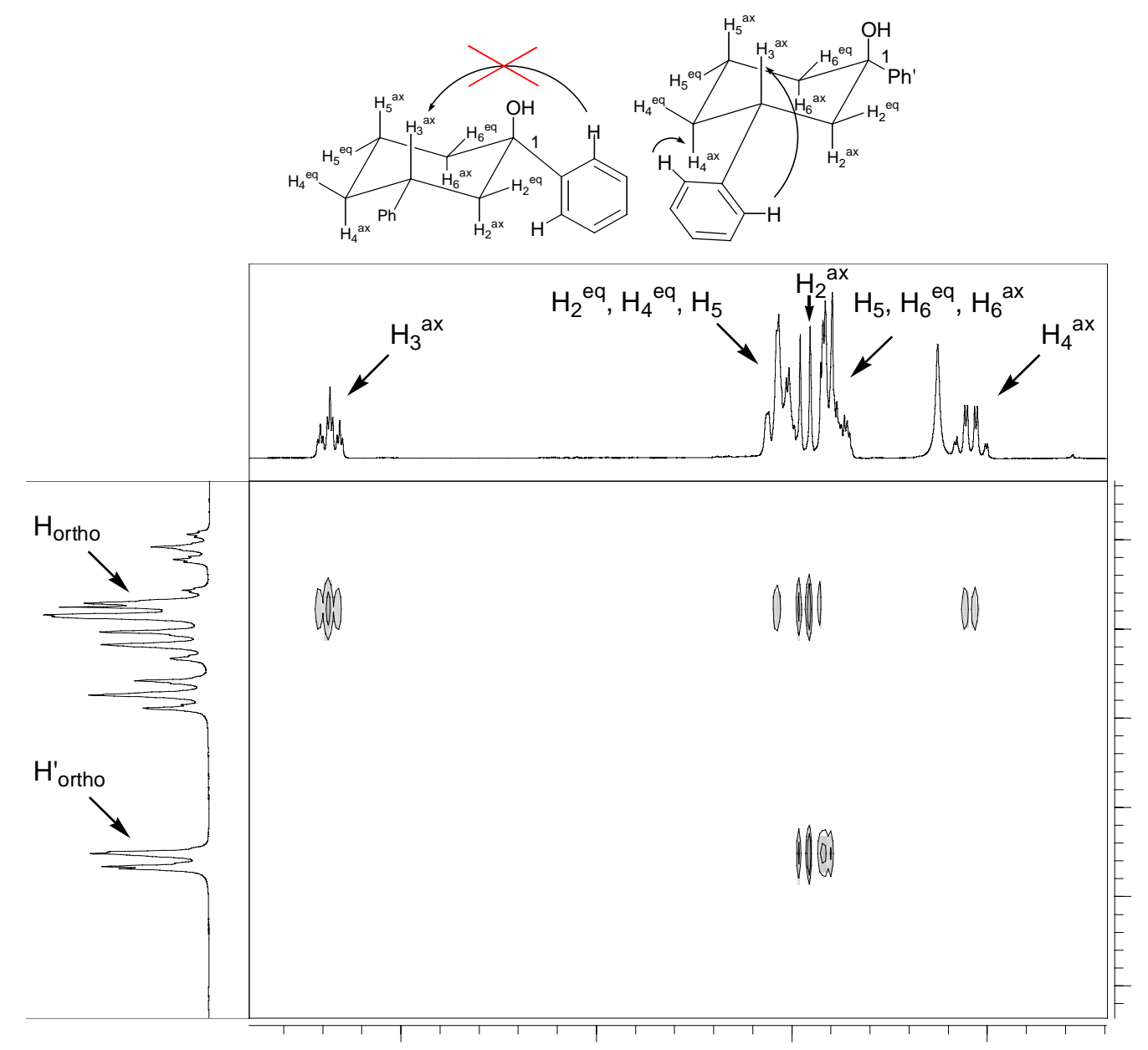

Figure 1 Arrows represent observed nOe signals between aromatic and aliphatic protons in (士)-14Aa

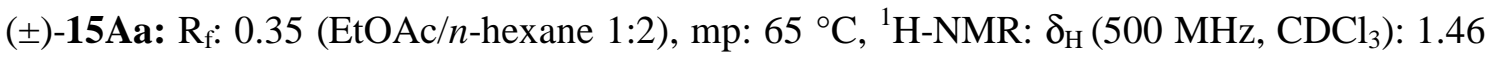
$\left(\mathrm{H}_{5}{ }^{\mathrm{ax}}\right.$, ddddd, $\left.\mathrm{J}=3.0,3.0,12.8,12.8,12.8 \mathrm{~Hz}\right), 1.50\left(\mathrm{H}_{4}{ }^{\mathrm{ax}}\right.$, dddd, 3.0, 12.8, 12.8, $\left.12.8 \mathrm{~Hz}\right)$, 1.74-1.93 $\left(\mathrm{H}_{4}{ }^{\mathrm{eq}}, \mathrm{H}_{5}{ }^{\mathrm{eq}}, \mathrm{H}_{6}{ }^{\mathrm{ax}}, \mathrm{OH}, \mathrm{m}\right), 1.99\left(\mathrm{H}_{2}{ }^{\mathrm{ax}}\right.$, dd (app. t), J = 12.8, $\left.12.8 \mathrm{~Hz}\right), 2.56\left(\mathrm{H}_{3}{ }^{\mathrm{ax}}\right.$, dddd, $\mathbf{J}=3.0,3.0,12.8,12.8 \mathrm{~Hz}), 2.63\left(\mathrm{H}_{6}{ }^{\mathrm{eq}}, \mathrm{dm}, \mathrm{J}=12.8 \mathrm{~Hz}\right), 2.72\left(\mathrm{H}_{2}{ }^{\mathrm{eq}}\right.$, ddt, $\mathbf{J}=1.8,3.0$, 3.0, $12.8 \mathrm{~Hz}), 7.19-7.24\left(2 \mathrm{H}_{\text {ortho }}, \mathrm{H}_{\text {para }}, \mathrm{m}\right), 7.29-7.36\left(2 \mathrm{H}_{\text {meta }}, \mathrm{H}_{\text {para }}, \mathrm{m}\right), 7.43\left(2 \mathrm{H}_{\text {meta }}, \mathrm{m}, \mathrm{J}=\right.$ $7.5 \mathrm{~Hz}), 7.61\left(2 \mathrm{H}^{\prime}{ }_{\text {ortho }}, \mathrm{m}, \mathrm{J}=7.5 \mathrm{~Hz}\right) \mathrm{ppm} .{ }^{13} \mathrm{C}-\mathrm{NMR}: \delta_{\mathrm{C}}\left(75.5 \mathrm{MHz}, \mathrm{CDCl}_{3}\right): 23.9\left(\mathrm{CH}_{2}\right)$, $34.0\left(\mathrm{CH}_{2}\right), 37.9\left(\mathrm{CH}_{2}\right), 41.5(\mathrm{CH}), 45.6\left(\mathrm{CH}_{2}\right), 74.0(\mathrm{C}), 126.2(\mathrm{CH}), 126.3(\mathrm{CH}), 126.8$ $(\mathrm{CH}), 127.6(\mathrm{CH}), 128.5(\mathrm{CH}), 128.8(\mathrm{CH}), 144.1(\mathrm{C}), 146.2(\mathrm{C}) \mathrm{ppm}$. MS m/z (rel. intensity \%). 252 (M+10), 234 (5), 209 (58), 133 (25), 120 (69), 105 (100), 91 (44), 77 (54). IR (KBr, thin film): 3559, 3366, 3086, 3060, 3026, 2935, 2861, 1602, 1492, 1450, 1387, 1332, 1294 , $1252,1197,1154,1070,1044,1028,952,922,768 \mathrm{~cm}^{-1}$. 


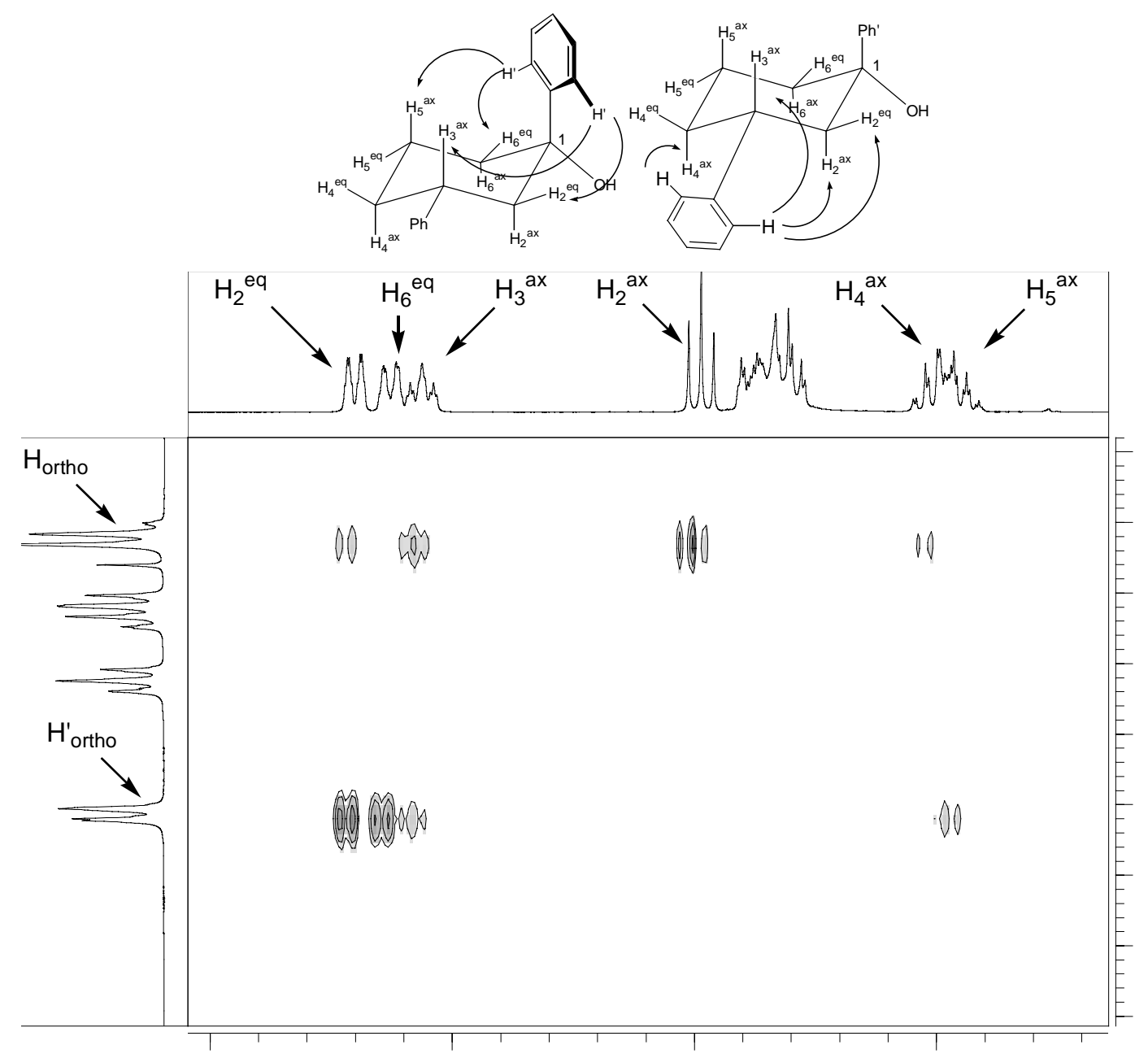

Figure 2. Arrows represent observed nOe signals between aromatic and aliphatic protons in ( \pm )-15Aa. The NMR signals of $\mathrm{H}_{6}{ }^{e q}$ and $\mathrm{H}_{2}{ }^{\text {eq }}$ show a downfield shift and the signals of $\mathrm{H}_{5}{ }^{a x}$ and $\mathrm{H}_{3}{ }^{a x}$ show an upfield shift relative to $( \pm)-14$ Aa.

The absolute configuration of (+)-14Aa (89\% ee) can be related to $(R)-\mathbf{1 3 A a}(91 \%$ ee), since its enantiomeric excess is almost equal to that of $(R)$-13Aa and a kinetic resolution favouring fast 1,2-addition to $(S)$-13Aa is not likely since the ee of $\mathbf{1 3 A a}$ is not substantially influenced by the amount of 14Aa. Moreover treatment of $19.0 \mathrm{mg}( \pm)-13 \mathrm{Aa}(0.109 \mathrm{mmol})$ with 5 equiv $\mathrm{PhB}(\mathrm{OH})_{2}$ and $3 \%(1 S, 4 S)$-catalyst prepared in situ resulted in $15.4 \mathrm{mg}$ of a mixture of $( \pm)$ 14Aa $(3 \%,<1 \%$ ee) and $( \pm)-13 A a(76 \%<1 \%$ ee $)$ indicating the absence of a kinetic resolution process capable of creating such a high enantiomeric excess in the formation of $(+)-\mathbf{1 4 A a}$ and therefore establishing the absolute configuration of $(+)-\mathbf{1 4 A a}$ to be $(1 R, 3 R)$. 

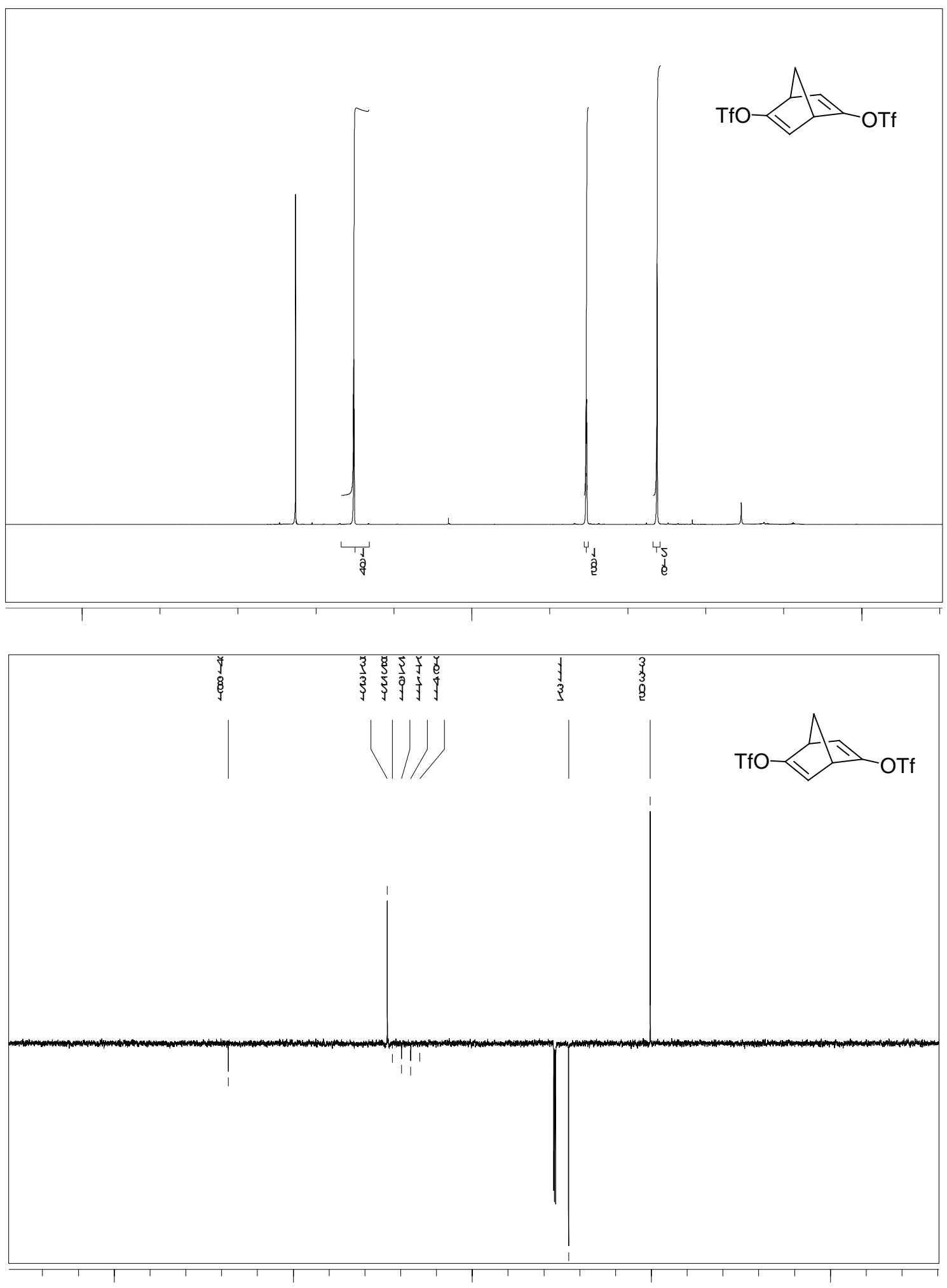

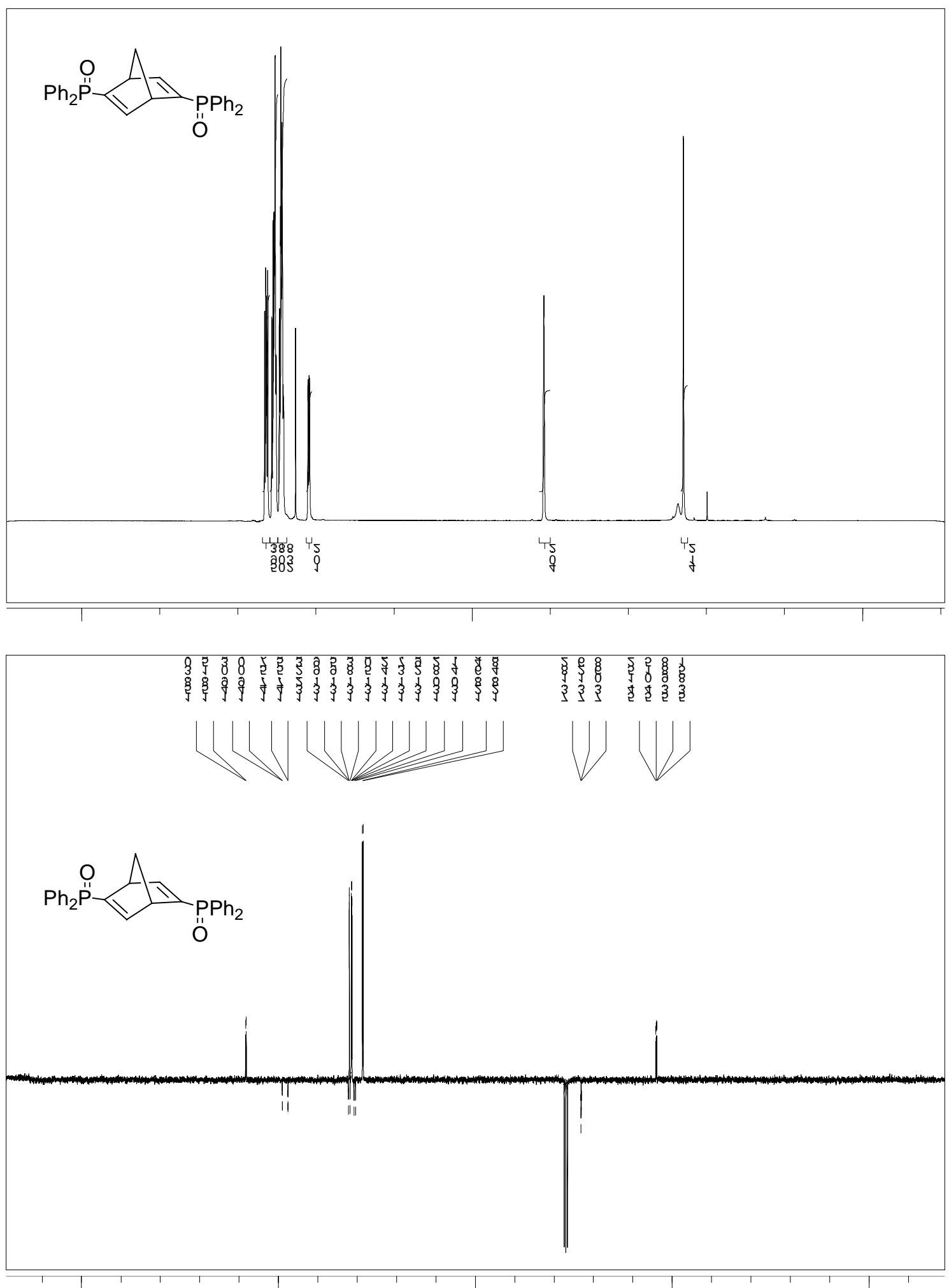

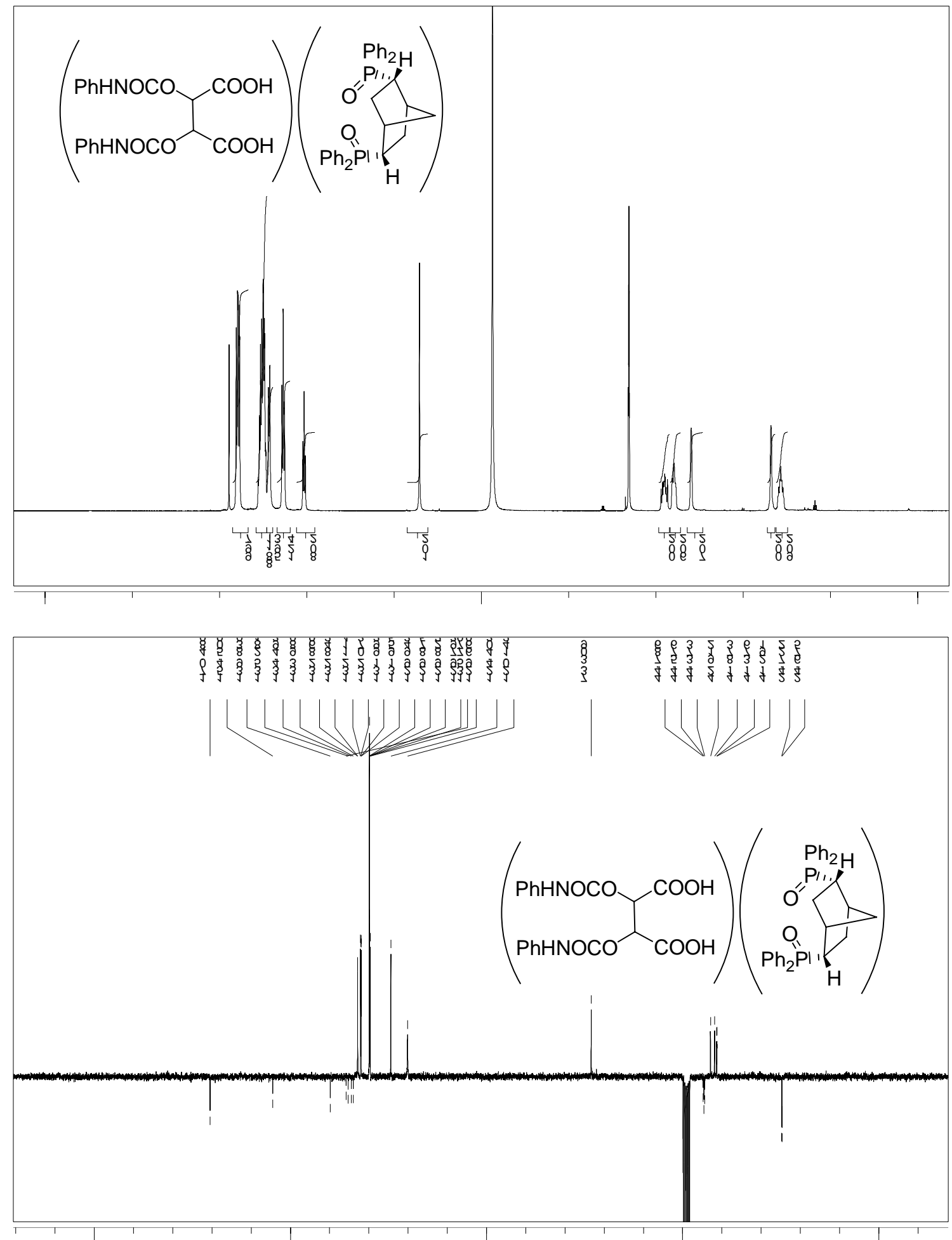

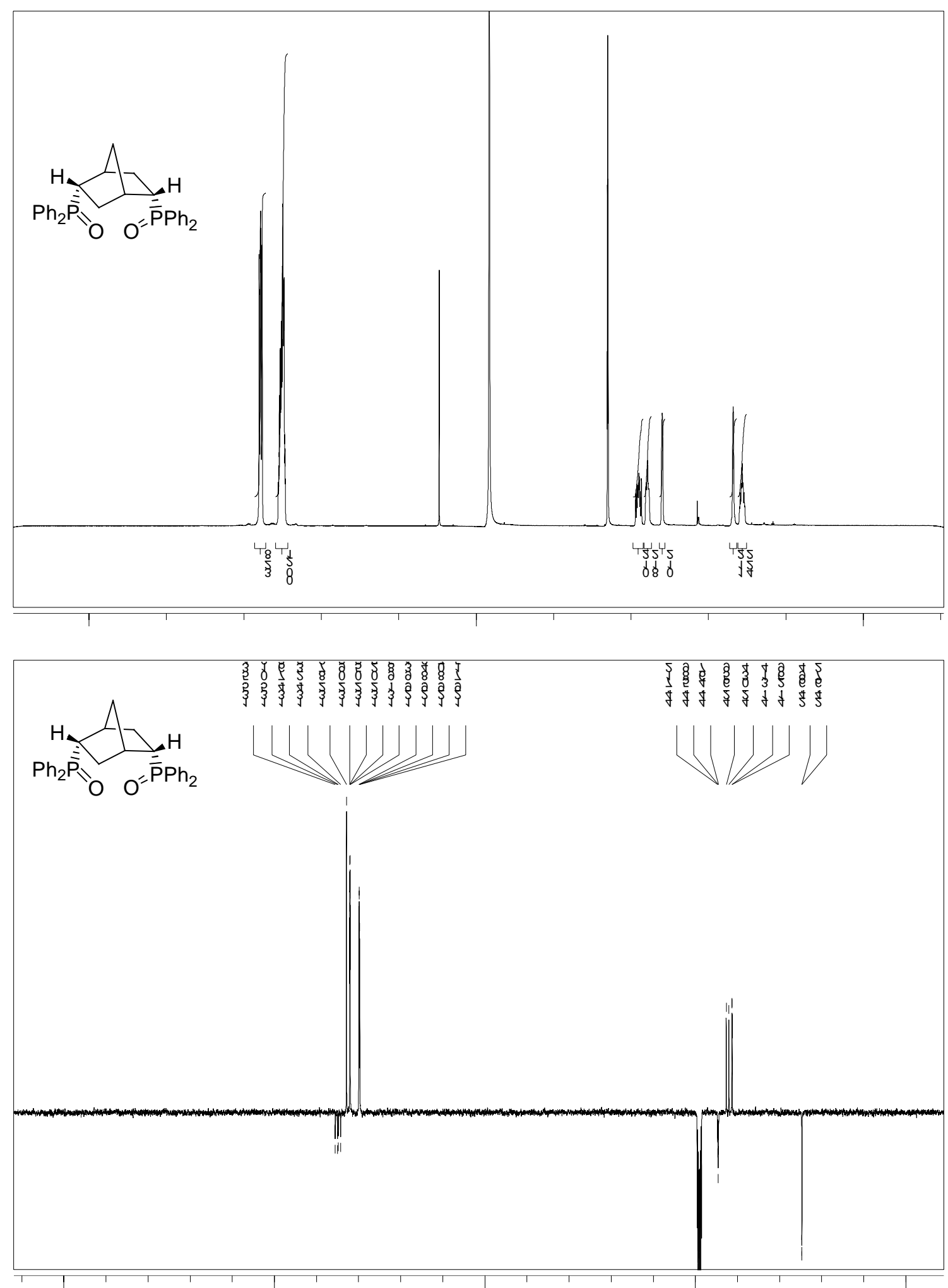

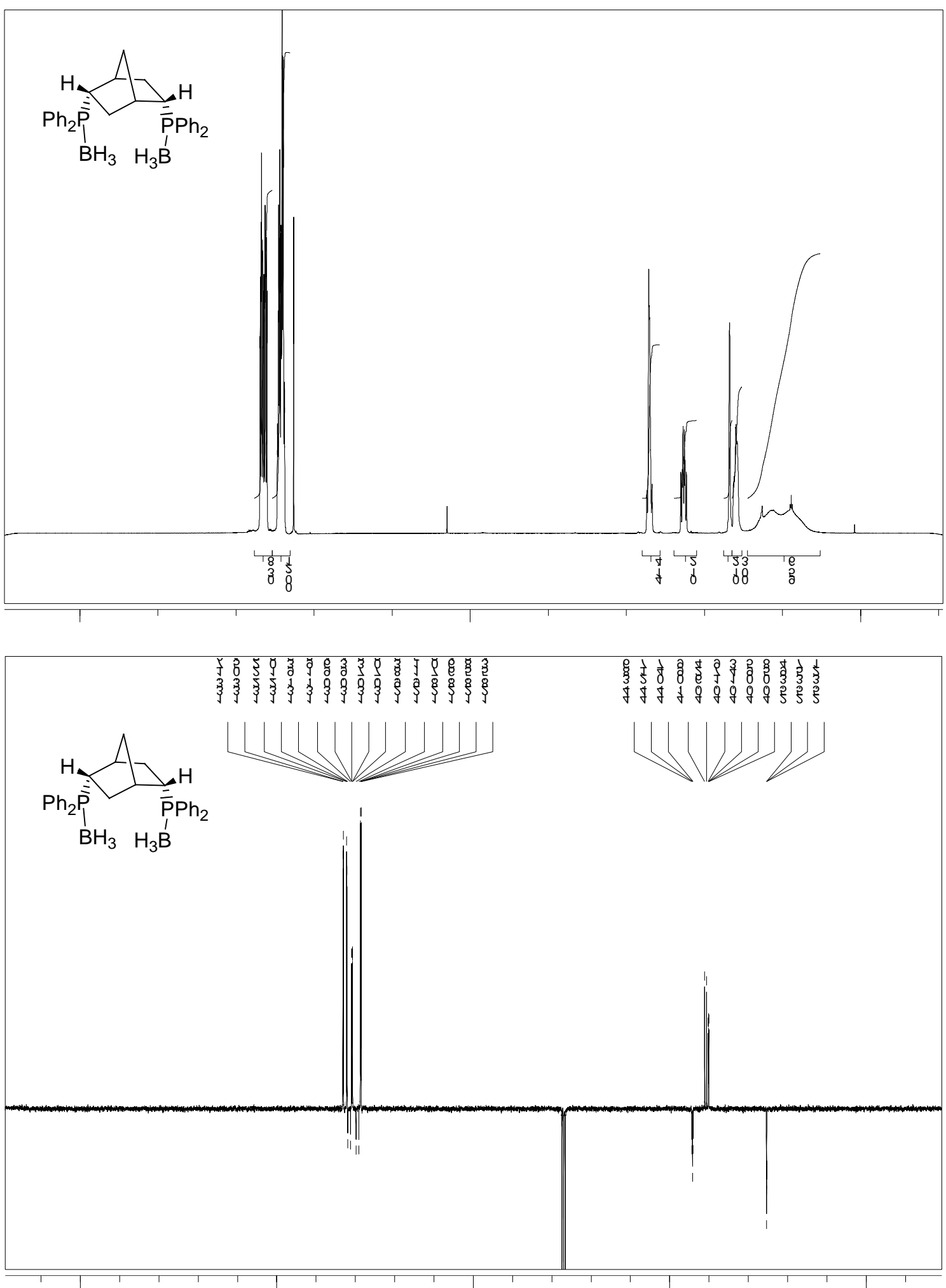

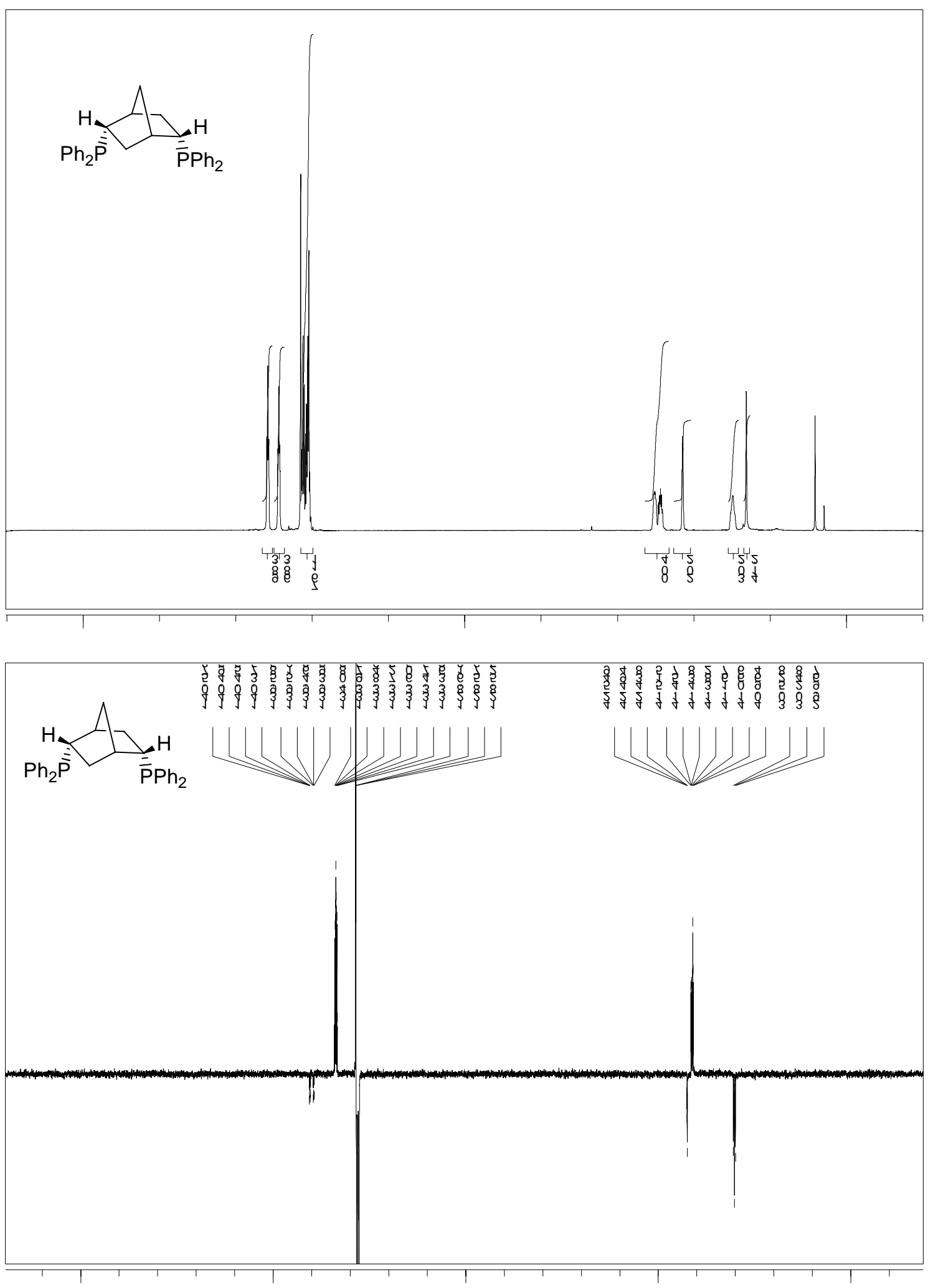

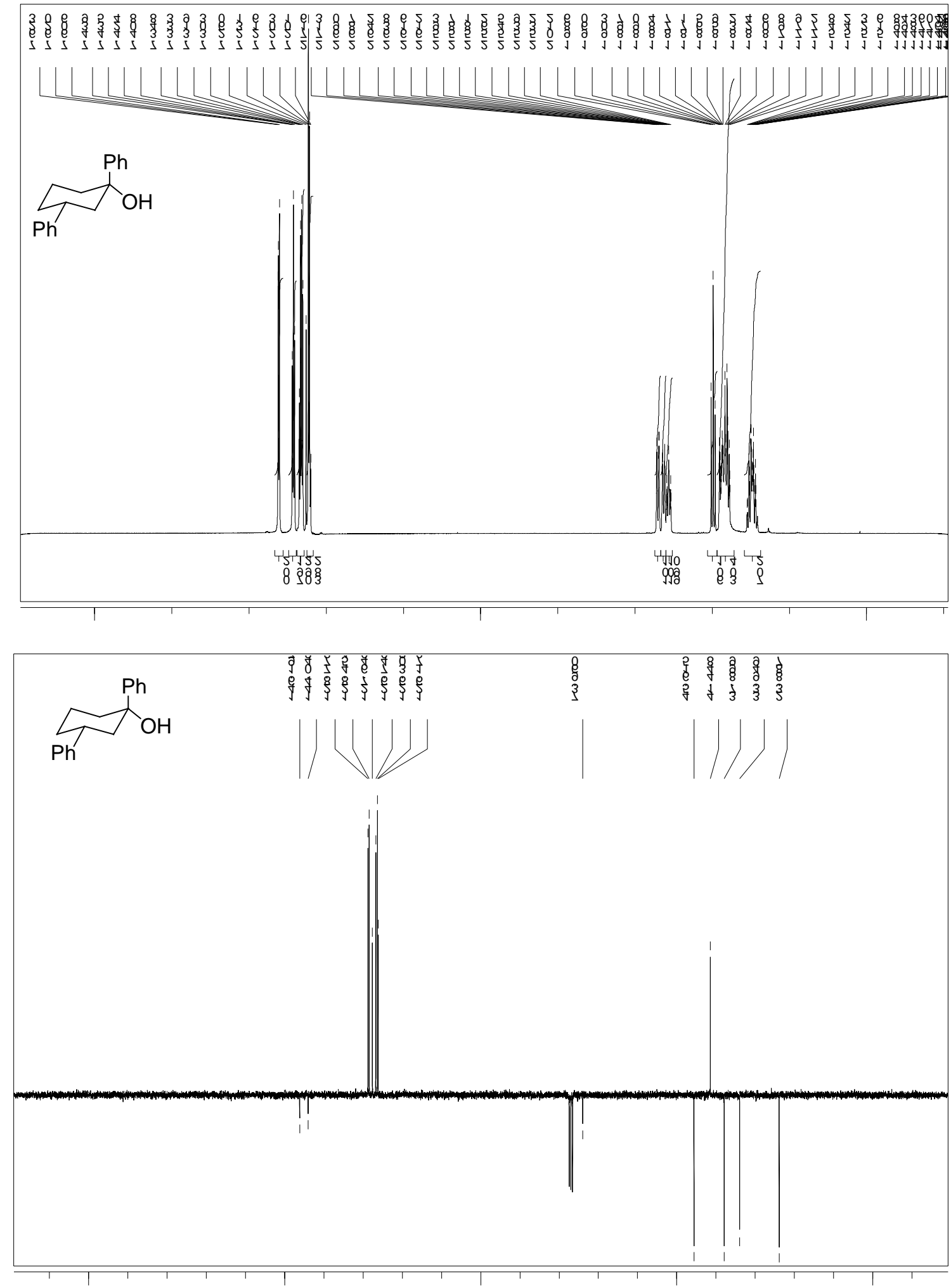

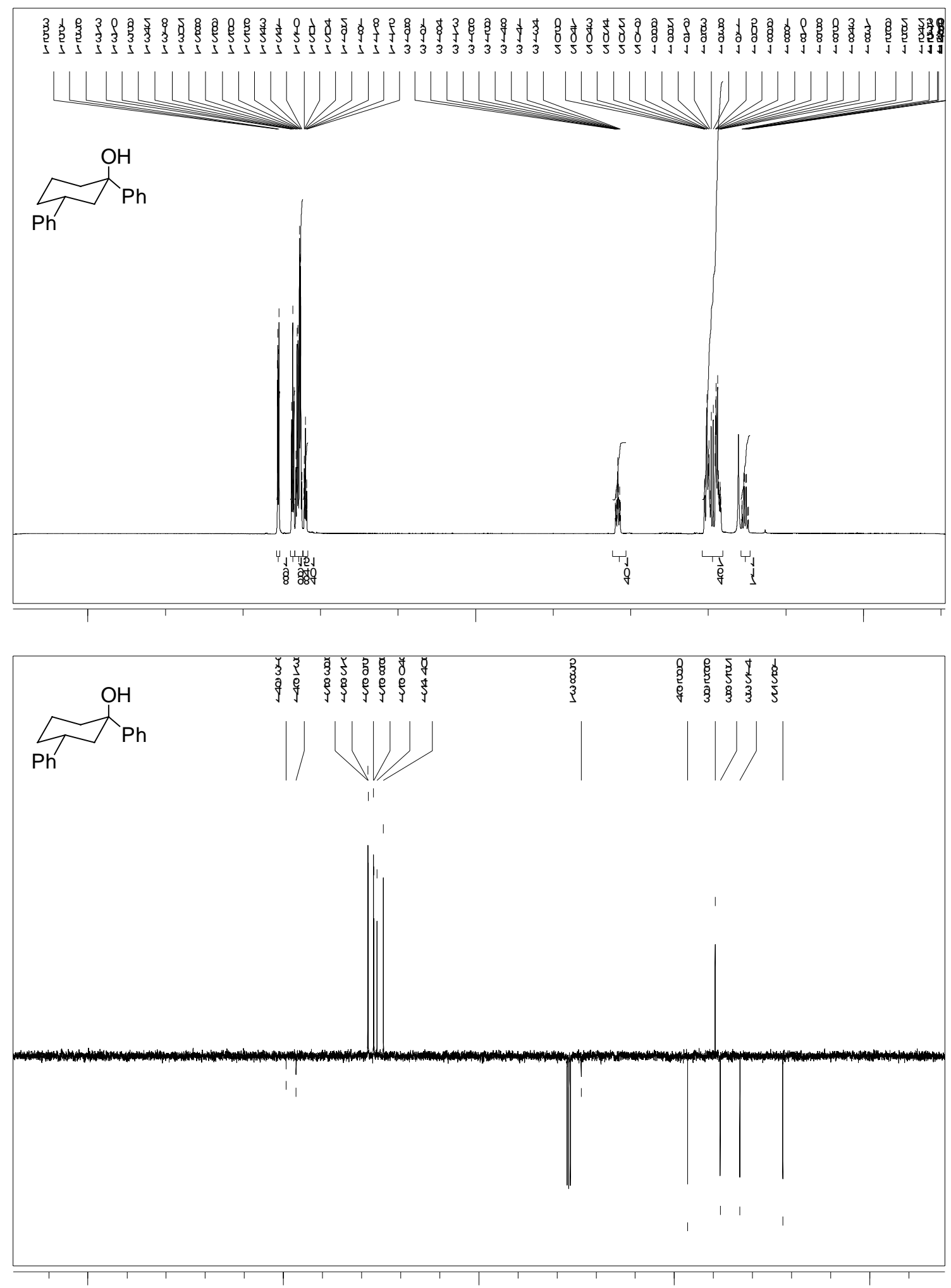

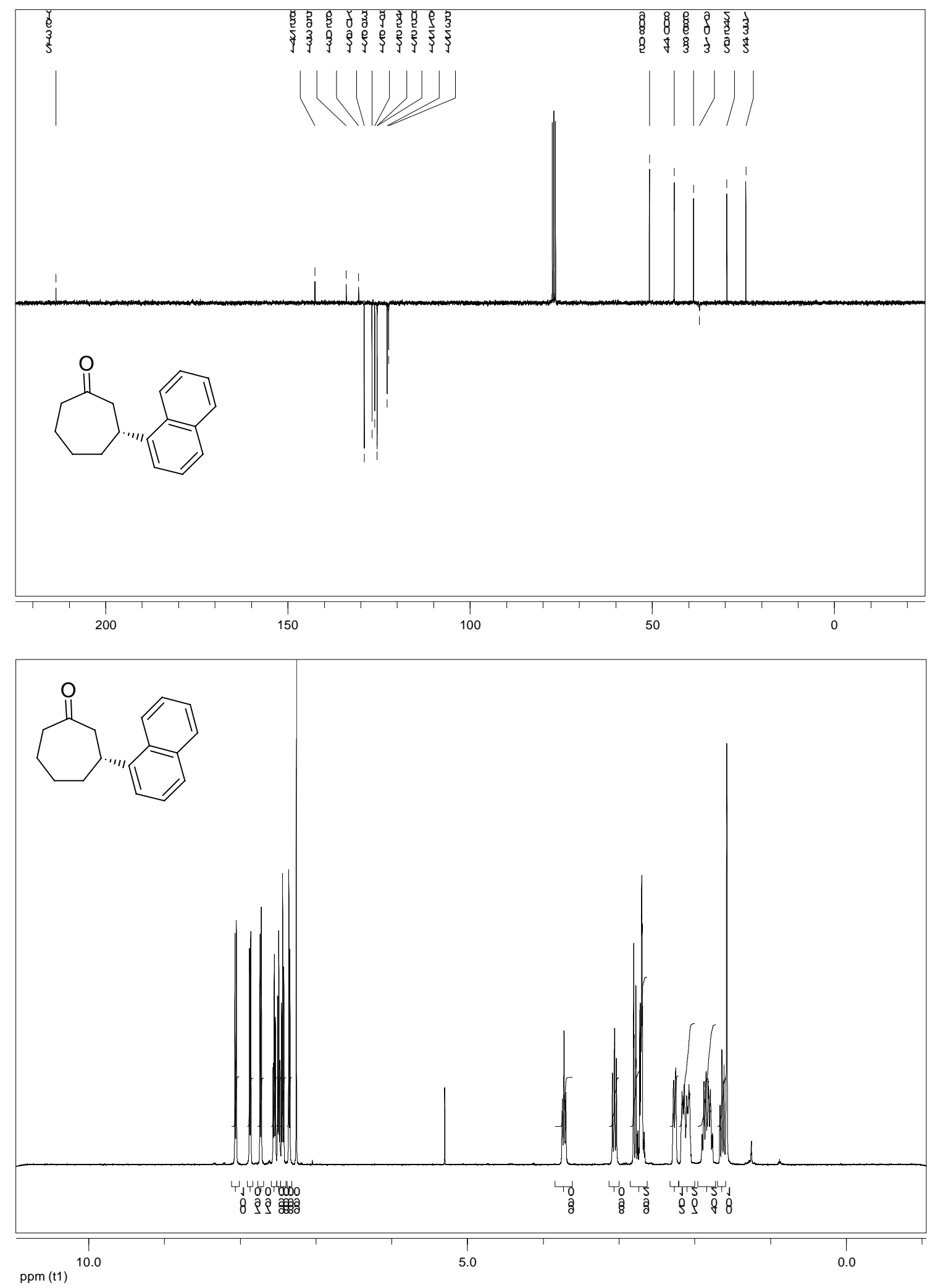

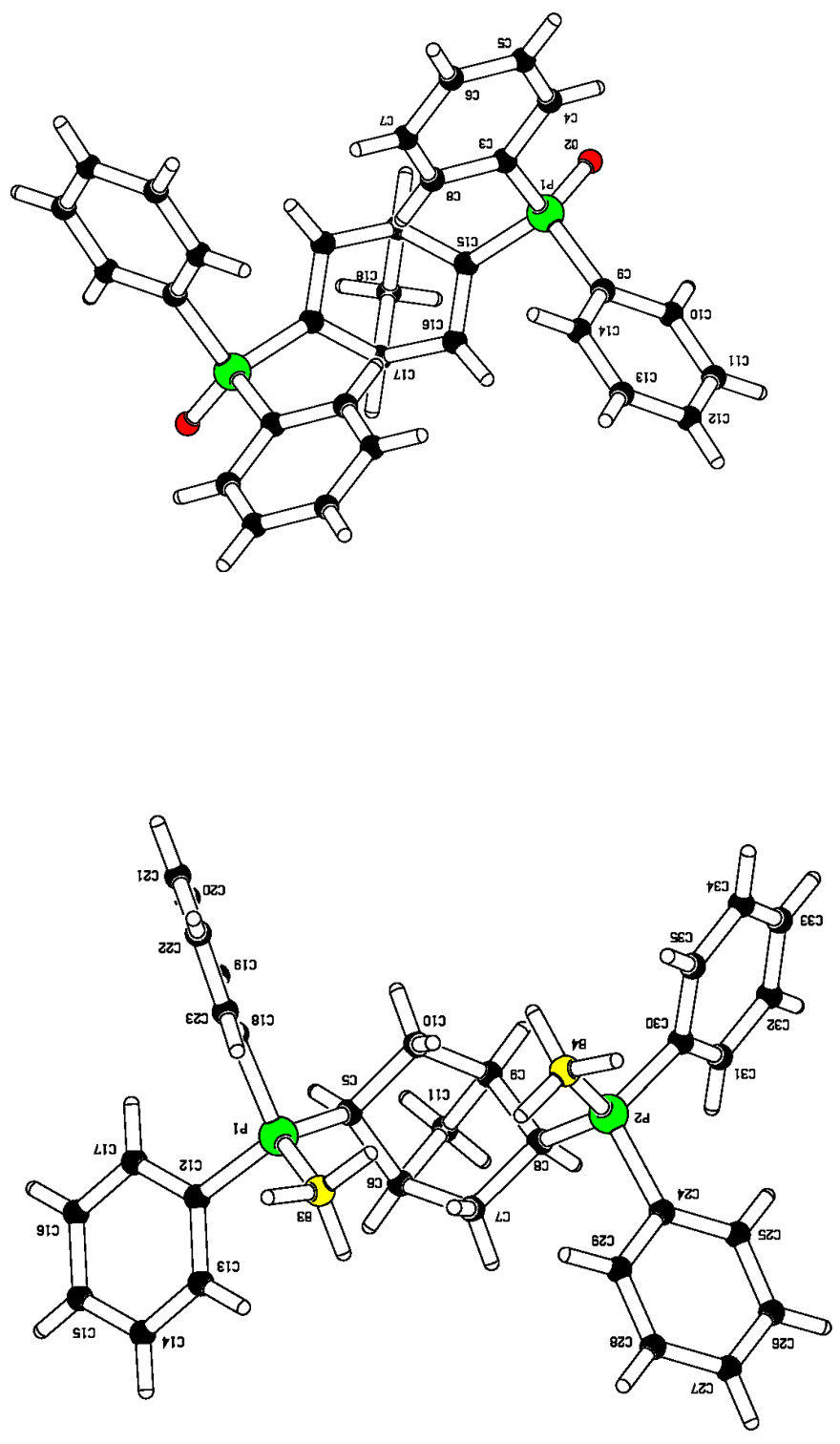